Ultrafast high strain rate acoustic wave measurements at high static pressure in a diamond anvil cell

M. Armstrong, J. Crowhurst, E. Reed, J. Zaug

February 6, 2008

Applied Physics Letters 
This document was prepared as an account of work sponsored by an agency of the United States government. Neither the United States government nor Lawrence Livermore National Security, LLC, nor any of their employees makes any warranty, expressed or implied, or assumes any legal liability or responsibility for the accuracy, completeness, or usefulness of any information, apparatus, product, or process disclosed, or represents that its use would not infringe privately owned rights. Reference herein to any specific commercial product, process, or service by trade name, trademark, manufacturer, or otherwise does not necessarily constitute or imply its endorsement, recommendation, or favoring by the United States government or Lawrence Livermore National Security, LLC. The views and opinions of authors expressed herein do not necessarily state or reflect those of the United States government or Lawrence Livermore National Security, LLC, and shall not be used for advertising or product endorsement purposes. 


\title{
Ultrafast high strain rate acoustic wave measurements at high static pressure in a diamond anvil cell
}

\author{
Michael R. Armstrong ${ }^{*}$, Jonathan C. Crowhurst, Evan J. Reed and Joseph M. Zaug \\ CSD/CMELS, Lawrence Livermore National Laboratory, P.O. Box 808; L-353, Livermore, CA \\ 94550
}

\begin{abstract}
We have used sub-picosecond laser pulses to launch ultra-high strain rate $\left(\sim 10^{9} \mathrm{~s}^{-1}\right)$ nonlinear acoustic waves into a 4:1 methanol-ethanol pressure medium which has been precompressed in a standard diamond anvil cell. Using ultrafast interferometry, we have characterized acoustic wave propagation into the pressure medium at static compression up to $24 \mathrm{GPa}$. We find that the velocity is dependent on the incident laser fluence, demonstrating a nonlinear acoustic response which may result in shock wave behavior. We compare our results with low strain, low strain-rate acoustic data. This technique provides controlled access to regions of thermodynamic phase space that are otherwise difficult to obtain.
\end{abstract}

*Author to whom correspondence should be addressed at armstrong30@1lnl.gov 
Material response to acoustic excitation is relevant to a broad spectrum of important topics including the interpretation of seismic waves, the composition and dynamics of planetary interiors, and fundamental questions in condensed matter physics ${ }^{1,2}$. Ultrafast acoustics have great potential for the investigation of material dynamics and chemical reactions with ultrafast strain modulation ${ }^{3-}$ 5. Studies at high initial pressure allow experiments to be initiated from a wide variety of initial thermodynamic states - near a phase transition for instance. ${ }^{1,6}$. Finally, ultrafast acoustic excitation occurs on a time and length scale that is directly accessible to molecular dynamics simulations. Here we use ultrafast interferometry to perform the first investigation of the dynamics of ultrafast high strain rate acoustic waves in a precompressed material.

In the present work, sub- $\mu \mathrm{J}, 800 \mathrm{~nm}$ wavelength, 800 fs duration pump pulses are tightly focused onto a $200 \mathrm{~nm}$ Al film which coats one inner face of a diamond anvil cell (DAC), as shown in Fig. 1, in which precompression $>100$ GPa may be obtained. Pump-induced thermal expansion over the deposition depth $(\sim 50 \mathrm{~nm})$ of the Al film launches an acoustic wave that travels through the film and enters a mixture of 4:1 methanol-ethanol. The velocity of the acoustic wave is measured by ultrafast time-resolved interferometry (Fig. 1) ${ }^{4,7,8}$. A 5 ps time-delayed probe and reference pair enter the DAC on the side opposite the pump at a variable time delay after the pump, allowing a pump induced phase shift to be measured ${ }^{4,8}$. Although ultrafast pump-probe techniques are well known in the study of acoustics ${ }^{4,5,7-9}$, to our knowledge this is the first application of ultrafast interferometry in a DAC.

Time series of phase shift vs. delay after pump arrival for various pressures are shown in Fig. 2. The data were obtained by measuring phase shifts for pump-probe delays in 5 ps increments, with 
40 shots per time delay. The total phase shift shown in Fig. 2 is the sum of all differential phase shifts up to the indicated time step. The data may be qualitatively divided into two contributions: a fast ( $<10 \mathrm{ps}$ ) rise between $40-50 \mathrm{ps}$ (corresponding to the arrival of the acoustic wave at the Al surface), followed by oscillatory variation of the phase that can be assigned to motion of the acoustic wave into the methanol-ethanol pressure medium.

The acoustic front modulates the index of refraction in the alcohol mixture. A scanning etalon between the acoustic front and the Al film results, which periodically modulates the referenceprobe phase difference. This signal is analogous to reflectivity (and more recently phase) modulation found in acoustic and shock wave measurements through transparent materials ${ }^{10,11}$ outside the DAC. To interpret the phase shift, we assume a reflected electric field of the form:

$$
A(t)\left[\begin{array}{c}
R_{s} \operatorname{Exp}\left[i\left(\phi_{s r}+\omega(t-\Delta t)\right)\right]+R_{f} \operatorname{Exp}\left[i\left(\phi_{f r}+\omega(t-\Delta t)\right)\right] \\
+R_{s} \operatorname{Exp}\left[i\left(\phi_{s p}+\omega(t+\Delta t)\right)\right]+R_{f} \operatorname{Exp}\left[i\left(\phi_{f p}+\omega(t+\Delta t)\right)\right]
\end{array}\right]
$$

where $A(t)$ is the electric field envelope of the probe and reference, the $\phi s$ are phase shifts associated with reference $(r)$ and probe $(p)$ reflections from the Al film $(f)$ and from the acoustic front $(s), R_{s, f}$ are the amplitudes of the electric field reflectivity from the acoustic front and film, and $\Delta \mathrm{t}$ is one half the delay between the probe and reference. A spectrum of the delayed pulses will be modulated at a frequency $\propto \frac{1}{\Delta t}$, and spectral oscillations at this frequency will exhibit a phase shift between pumped and unpumped regions of the probe. The measured differential phase may be integrated to give:

$$
\phi=\phi_{f}-\frac{R_{s}}{R_{f}} \sin \left(\phi_{f}-\phi_{s}\right)
$$


for the total phase shift, where $\phi_{f}-\phi_{s}$ is related to acoustic wave motion with respect to the Al interface, $\phi_{f}$ is related to the motion of the $\mathrm{Al}$ interface and all phases are functions of time. Essentially, the measured phase at a given time delay is a finite derivative over the $5 \mathrm{ps}$ probe/reference delay, which may be integrated to give a profile with the form of eq. 1 .

The acoustic wave velocity through the pressure medium is given by $\frac{\lambda}{2 n \tau_{p}}$, where $\lambda$ is the probe wavelength, $n$ is the index of the material between the acoustic front and the $\mathrm{Al}$ interface, and $\tau_{\mathrm{p}}$ is the period of the phase oscillation ${ }^{10}$. For the pressure dependent index, we assume static values measured by Eggert et al. ${ }^{12}$

Error in the absolute velocity measurement comprises statistical error, absolute error resulting from the use of the static index of refraction, and error arising from a dependence of velocity on the pump fluence. The standard deviation of the frequency estimate is given by the Cramer-Rao lower bound ${ }^{13}$, in this case less than $+/-1 \%$ for the worst signal to noise traces. An absolute determination of the velocity depends on the index of the material between the acoustic front and the Al film, leading to error if the index between the acoustic front and the $\mathrm{Al}$ film differs from the static value. A bound on this error may be estimated via the amplitude of the oscillatory term in eq. $1\left(\frac{R_{s}}{R_{f}}\right)$, since $R_{s}$ is related to the index change at the acoustic front and $R_{f}$ is near 1 . In the most extreme case - a shock wave (where the entire region downstream has an elevated index) $-R_{s}$ is approximately $\frac{\Delta n}{2 n}$ corresponding to Fresnel reflection at a step index boundary. The maximum amplitude of the measured oscillations is less than $10 \mathrm{mrad}$, which would give an index modulation 
at the boundary of less than $3 \times 10^{-2}\left(2 \mathrm{n} \mathrm{R}_{\mathrm{s}}\right)$. This would result in a $2 \%$ overestimate of the velocity given by the unmodulated refractive index. Assuming the index change downstream from the acoustic front is not sustained, this error estimate for velocity variation owing to an inaccurate index estimate downstream is quite conservative. More generally, when applied to transparent materials at very high strain in shock wave experiments, this method and analogous methods (like VISAR) must accurately account for this effect ${ }^{14}$. Finally, measurements of velocity as a function of pump fluence (shown in Fig. 4 and discussed below) show a variation of the measured velocity approximately $2 \%$ over the fluence range tested. We conservatively estimate a maximum variation of $2 \%$ in the velocity measurements owing to misalignment between the pump and probe between pressure adjustments in the DAC and variation in pump energy. Errors in the index estimate will overestimate the velocity and errors in the alignment will underestimate the velocity. Given these error estimates, we assign error bars of $+/-3 \%$ to all velocity measurements.

Fig. 3 presents pressure dependent velocities up to $24 \mathrm{GPa}$ precompression. For comparison to low strain, lower frequency sound speeds $(\sim 1 \mathrm{GHz})$, the plot also shows sound speeds in pure methanol (measured by Zaug et al. ${ }^{15}$ ) and 4:1 ethanol-methanol, both using impulsive stimulated light scattering (ISLS). Brown et al. give the same velocities for pure ethanol and pure methanol as recorded by Zaug et al. up to $3 \mathrm{GPa}$ with a cited error of $1 \%{ }^{16}$. The speeds measured using ultrafast interferometry are equal to or larger than the low strain, low frequency sound speed in pure methanol and 4:1 methanol-ethanol. Zaug et al. observed variation in the sound speed of pure methanol at $3.7 \mathrm{GPa}$ as a function of excitation frequency, which suggests that acoustic dispersion may in part account for the higher acoustic velocities we measure. The excitation frequency used in 
our 4:1 methanol-ethanol ISLS measurement is $\sim 2 \mathrm{x}$ that used in the methanol measurements of Zaug et al.

Velocity measurements as a function of pump fluence (Fig. 4) indicate that the pressure medium exhibits a nonlinear response to strain. As the pump energy is increased, the measured velocity increases. The error bars on the data in Fig. 4 are $+/-1 \%$, smaller than the error bars in fig. 3 because these fluence dependent measurements are only meant to demonstrate a relative change in the velocity with pump fluence and pump power can be varied with no realignment (in contrast to static pressure variation, which requires the DAC to be removed and replaced), so error due to index estimation and static pressure modulation is not included.

The amplitude of the phase variations $\left(\frac{R_{s}}{R_{f}}\right)$ for pressures between 3-15 GPa ranges from 5-8 mrad. Assuming a step index change (as described above) at the acoustic front, the reflection amplitude would give $\Delta \mathrm{n}$ between $1.5-2.5 \times 10^{-2}$ (i.e. $\Delta n=2 n R_{s}$, here we assume $\mathrm{R}_{\mathrm{f}} \sim 1$ ). At $11 \mathrm{GPa}$, the static index of refraction vs. pressure given by Eggert et al. ${ }^{12}$ give the variation of index with pressure as $9.4 \times 10^{-3} / \mathrm{GPa}$. Assuming this variation of index with pressure, the phase amplitude of $8 \mathrm{mrad}$ at 11 GPa implies an index difference of $\sim 2.6 \times 10^{-2}$ across the acoustic front giving a transient pressure of $2.8 \mathrm{GPa}$ at $11 \mathrm{GPa}$ static pressure. Using the equation of state estimated by Eggert et al. ${ }^{12}$, a pressure difference in this range implies a strain of approximately $1 \%$ and a strain rate (for a rise that is $<10 \mathrm{ps}$ ) greater than $10 \%$ s. This is an estimate of the strain at the acoustic front based on the reflectivity $\left(\mathrm{R}_{\mathrm{s}}\right)$. Strain downstream from the acoustic front is likely smaller. Nonetheless, the amplitude of phase oscillations does not significantly decay, implying that strain at the acoustic front does not significantly change. A more conservative assumption of a pulse excitation at the 
acoustic front yields half the index change given by a step modulation (i.e. $\Delta n=n R_{s}$ ), and this reduces our estimate of transient pressure to $1.4 \mathrm{GPa}$ (at $11 \mathrm{GPa}$ static precompression). Using these two estimates as upper and lower bounds, the change in pressure across the acoustic front corresponds to a $5-10 \%$ change in sound speed across the acoustic front. This speed modulation across the acoustic front may exhibit shock wave characteristics. At $11 \mathrm{GPa}$, the sound speed is $\sim 7$ $\mathrm{km} / \mathrm{s}$. Assuming a spatial extent of $<70 \mathrm{~nm}$ for the acoustic front (corresponding to $<10 \mathrm{ps}$ initial drive duration), a $5 \%$ velocity modulation across a step rise in strain will cause the wavefront to steepen to a discontinuity in strain (forming a shock wave) within $200 \mathrm{ps,}$ or 1.4 microns from the Al launch surface, much less than the diameter of the excitation region $(20 \mu \mathrm{m})$.

In conclusion, we have directly measured the velocity of high strain rate ultrafast acoustic fronts under high static pressure in a diamond anvil cell. The acoustic velocity of 4:1 ethanol-methanol measured using ultrafast interferometry at high strain rate is equal to or larger than the low strain rate sound speed of methanol and 4:1 methanol-ethanol. Furthermore, the sound speed at high strain rate exhibits a dependence on pump energy. Although high precision data on acoustic wave speeds in $\mathrm{Al}$ as a function of static pressure were not obtained in this work, in principle it is possible to measure the time of flight in bulk metals along the DAC axis, and our data demonstrate the feasibility of this proposal. These measurements were performed in a conventional DAC, enabling measurements starting at high static pressure, potentially up to $100 \mathrm{GPa}$.

A significant consequence of the demonstrated nonlinear acoustic response is the new experimental capability to generate and probe previously inaccessible high $\mathrm{p}, \mathrm{T}$ thermodynamic regimes of materials. For example, ramp and shock wave compression in the DAC can generate states nearer 
to the cores of giant planets than any other technique ${ }^{6}$. Our results demonstrate the experimental potential to generate such waves. The ability to tune the initial thermodynamic state of a material before applying an ultrafast strain will significantly expand the scope and versatility of investigations into strain induced chemistry ${ }^{3}$ and ultrafast phase transitions ${ }^{17}$.

The authors would like to thank A. Bliss, C. A. Bolme, J. M. Brown, L. E. Fried, D. J. Funk, C. D. Grant, W. M. Howard, A. Goncharov, E. Lee, D. S. Moore, K. A. Nelson, C. Tarver, and B. R. Torralva. This work was supported by LLNL LDRD project 06-SI-005 and was performed under the auspices of the U.S. Department of Energy by Lawrence Livermore National Laboratory under Contract DE-AC52-07NA27344. 


\section{References}

$1 \quad$ W. J. Nellis, Rep. Prog. Phys. 69 (5), 1479-1580 (2006).

$2 \quad$ W. J. Nellis, J. Phys.-Condes. Matter 14 (44), 11045-11054 (2002).

3 J. E. Patterson, A. Lagutchev, W. Huang, and D. D. Dlott, Phys. Rev. Lett. 94 (1) (2005).

$4 \quad$ K. T. Gahagan, D. S. Moore, D. J. Funk, R. L. Rabie and S. J. Buelow, Phys. Rev. Lett. 85 (15), 3205-3208 (2000).

$5 \quad$ R. M. Slayton and K. A. Nelson, J. Chem. Phys. 120 (8), 3919-3930 (2004).

R. Jeanloz, P. M. Celliers, G. W. Collins, J. H. Eggert, K. K. M. Lee, R. S. McWilliams, S.

Brygoo and P. Loubeyre, PNAS 104 (22), 9172-9177 (2007).

R. Evans, A. D. Badger, F. Fallies, M. Mahdieh, T. A. Hall, P. Audebert, J.-P. Geindre, J.-C.

Gauthier, A. Mysyrowicz, G. Grollon and A. Antonetti, Phys. Rev. Lett. 77 (16), 3359-3362 (1996).

J. P. Geindre, P. Audebert, A. Rousse, F. Fallies, J. C. Gauthier, A. Mysyrowicz, A.

Dossantos and G. Hamoniaux, Opt. Lett. 19 (23), 1997-1999 (1994).

D. J. Funk, D. S. Moore, K. T. Gahagan, S. J. Buelow, J. H. Reho, G. L Fisher and R. L.

Rabie, Phys. Rev. B 6411 (11) (2001)

C. J. Stanton, G. D. Sanders, R. Liu, G. W. Chern, C. K. Sun, J. S. Yahng, Y. D. Jho, J. Y.

Sohn, E. Oh and D. S. Kim, Superlattices Microstruct. 34 (3-6), 525-529 (2003). (2007). 2461 (1992).

David C. Rife and Robert R. Boorstyn, IEEE T Inform Theory 20 (5), 591-598 (1974). 
R. E. Setchell, J. Appl. Phys. 91 (5), 2833-2841 (2002).

J. M. Zaug, L. J. Slutsky, and J. M. Brown, J. Phys. Chem. 98 (23), 6008-6016 (1994).

16

J. M. Brown, L. J. Slutsky, K. A. Nelson and L.-T. Cheng., Science 241 (4861), 65-67 (1988).

17 B. J. Siwick, J. R. Dwyer, R. E. Jordan and R. J. D. Miller, Science 302 (5649), 1382-1385 (2003). 


\section{Figure captions}

Figure 1: A close up of the DAC with pump, probe and reference pulses. The probe optical wavefronts are phase shifted where the probe overlaps the pump due to strain-induced displacement of the Al surface and a scanning etalon in the pressure medium. The pump is focused to a $20 \mu \mathrm{m}$ spot size.

Figure 2: The integrated phase shift for experiments at pressures up to $24.1 \mathrm{GPa}$. The plotted total phase shift is the sum of all measured phase shifts obtained up to the indicated time step. Fits to the oscillatory part of the signal are shown as dashed lines. The oscillations are fit to a linear offset plus a sinusoidal oscillation. Also, the acoustic wave transit time in Al decreases with increasing pressure, corresponding to increasing speed.

Figure 3: Ultrafast and ISLS sound speed measurements in 4:1methanol-ethanol and methanol. The following pump pulse energies were used for the ultrafast measurement: $0-7.4 \mathrm{GPa}, 0.4 \mu \mathrm{J} ; 8-24.1$ GPa $0.6 \mu \mathrm{J}$. The errors in the ISLS measurements are $+/-0.2 \%$ and $+/-1 \%$ for methanol (Zaug et al.) and 4:1 ethanol-methanol, respectively.

Figure 4: Acoustic speed as a function of pulse energy at static compressions of $19.3 \mathrm{GPa}$ (top) and 7.4 GPa (bottom). For $1 \mu \mathrm{J}$ energy in the $7.4 \mathrm{GPa}$ data, some sample damage was observed. The 7.4 GPa data at lower energy were taken in the sequence: $0.2 \mu \mathrm{J}, 0.5 \mu \mathrm{J}, 0.8 \mu \mathrm{J}, 0.5 \mu \mathrm{J}$, with the higher speed data point at $0.5 \mu \mathrm{J}$ taken second. 


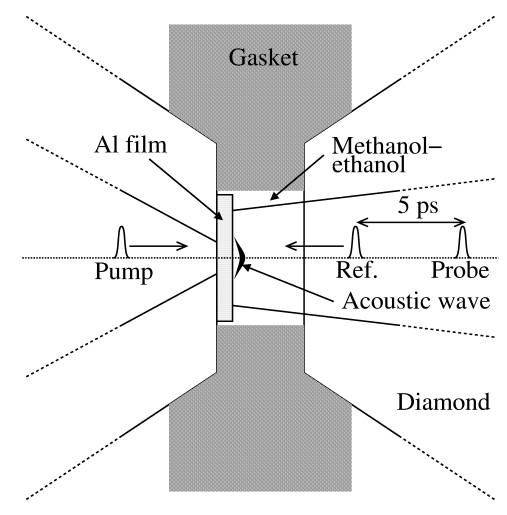

Figure 1: A close up of the DAC with pump, probe and reference pulses. The probe optical wavefronts are phase shifted where the probe overlaps the pump due to strain-induced displacement of the Al surface and a scanning etalon in the pressure medium. The pump is focused to a $20 \mu \mathrm{m}$ spot size. 


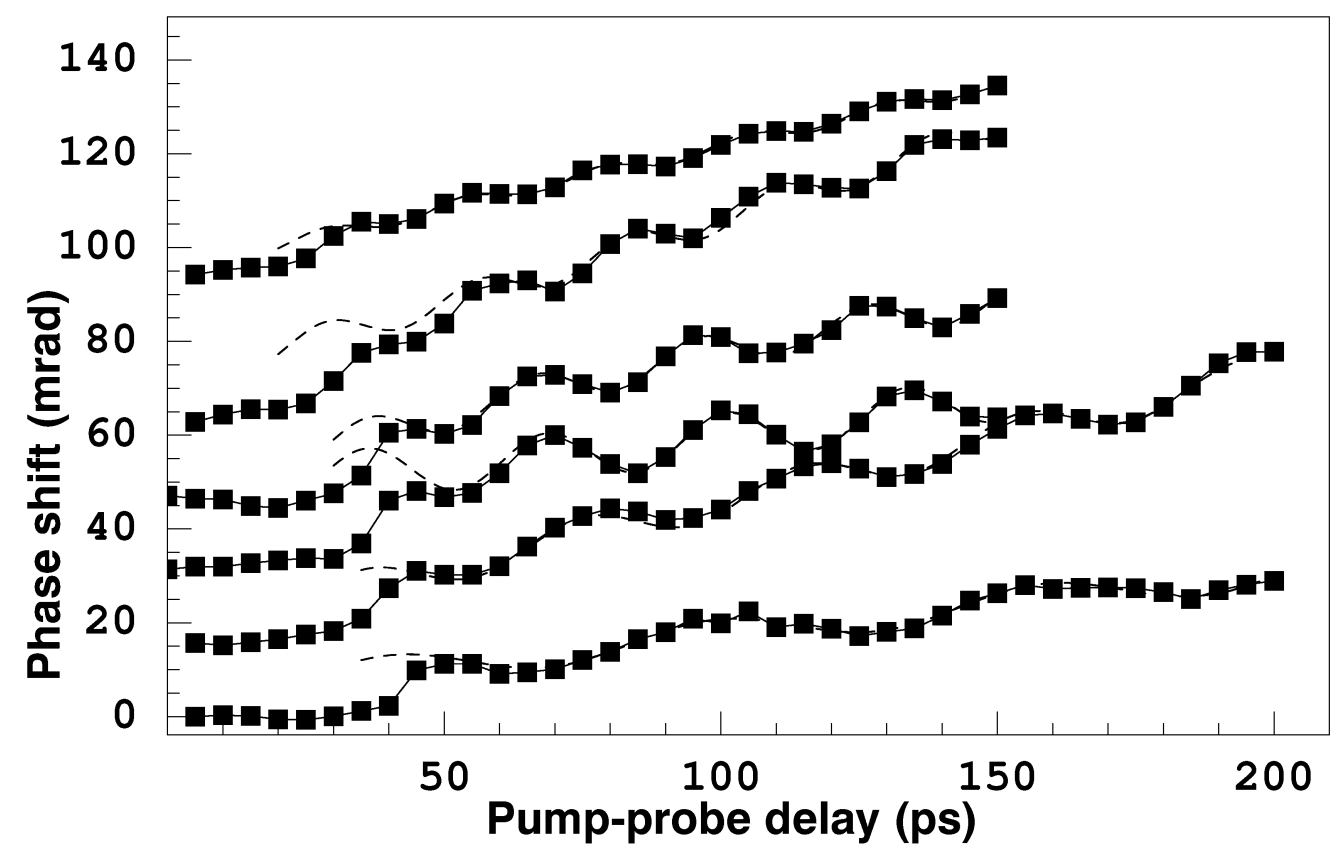

Figure 2: The integrated phase shift for experiments at pressures up to $24.1 \mathrm{GPa}$. The plotted total phase shift is the sum of all measured phase shifts obtained up to the indicated time step. Fits to the oscillatory part of the signal are shown as dashed lines. The oscillations are fit to a linear offset plus a sinusoidal oscillation. Also, the acoustic wave transit time in Al decreases with increasing pressure, corresponding to increasing speed. 


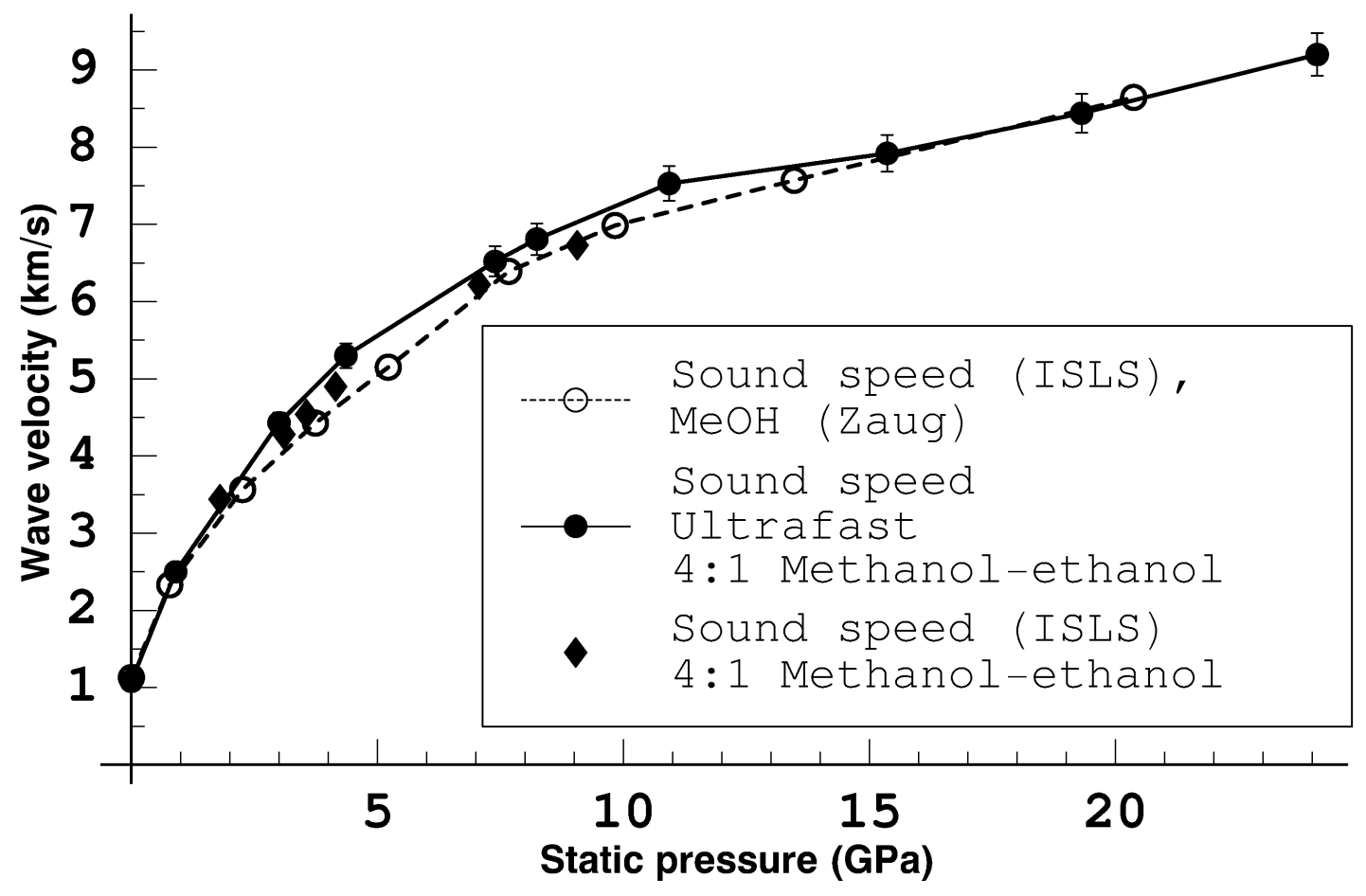

Figure 3: Ultrafast and ISLS sound speed measurements in 4:1methanol-ethanol and methanol. The following pump pulse energies were used for the ultrafast measurement: 0-7.4 GPa, $0.4 \mu \mathrm{J} ; 8$-24.1 GPa 0.6 $\mu \mathrm{J}$. The errors in the ISLS measurements are $+/-0.2 \%$ and $+/-$ $1 \%$ for methanol (Zaug et al.) and 4:1 ethanol-methanol, respectively. 


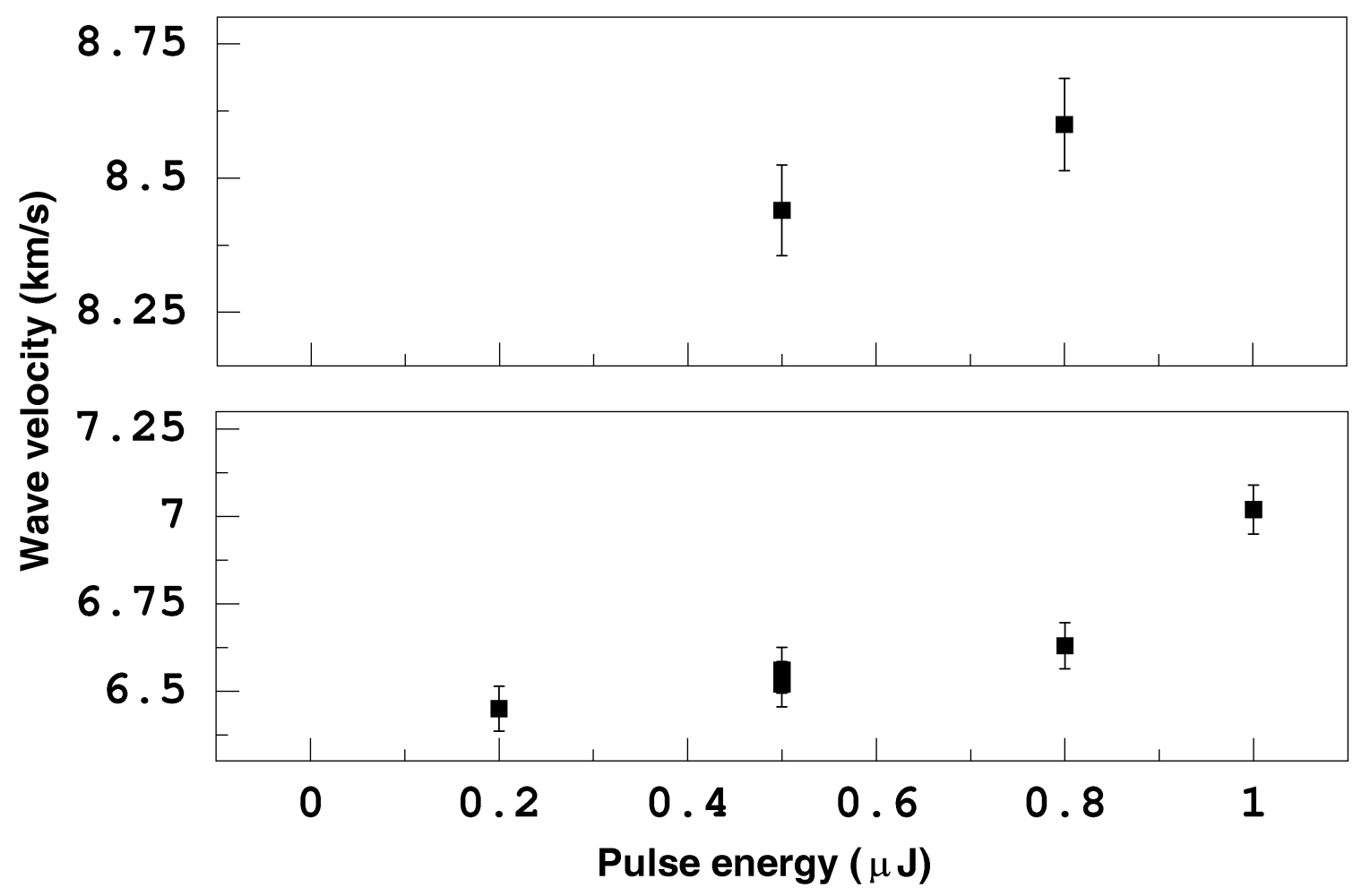

Figure 4: Acoustic speed as a function of pulse energy at static compressions of 19.3 GPa (top) and 7.4 GPa (bottom). For $1 \mu \mathrm{J}$ energy in the 7.4 GPa data, some sample damage was observed. The 7.4 GPa data at lower energy were taken in the sequence: $0.2 \mu \mathrm{J}, 0.5 \mu \mathrm{J}, 0.8 \mu \mathrm{J}, 0.5 \mu \mathrm{J}$, with the higher speed data point at $0.5 \mu \mathrm{J}$ taken second. 